\title{
Sensitization of Gastric Cancer Cells to 5-FU by MicroRNA-204 Through Targeting the TGFBR2-Mediated Epithelial to Mesenchymal Transition
}

\author{
Liang-Qing Li Dun Pan Qun Chen Sheng-Wei Zhang Di-Ya Xie \\ Xue-Lan Zheng Hui Chen
}

Department of gastrointestinal surgery, The First Affiliated Hospital of Fujian Medical University, Fuzhou, China

\section{Key Words}

MicroRNA-204 •5-FU • Chemoresistance - TGFBR2 • Epithelial to mesenchymal transition

\begin{abstract}
Background/Aims: Gastric cancer (GC) is the most common gastrointestinal malignancy, causing cancer-related deaths in East Asia. MicroRNAs (miRNAs) are small non-coding RNAs aberrantly expressed in human tumors. In this study, we aim to investigate the roles of miR-204 in the epithelial to mesenchymal transition (EMT)-associated chemosensitivity. Methods: The expression of miR-204 was detected in clinical tumor samples and GC cell lines by real time PCR. Tumor cell's growth, invasion, and migration were measured by MTT assay, wound healing assay, and transwell invasion assay, respectively. Western blot method was used to detect the protein levels of indicated genes. Luciferase reporter assay was performed to validate the target gene of miR-204. The in vivo role of miR-204 was measured using a xenograft mouse model of GC. Results: By comparing the expressions of miR-204 in human gastric tumors and their adjacent normal tissues, it was disclosed that miR-204 was significantly downregulated in gastric tumors. Moreover, miR-204 was downregulated in multiple GC cell lines compared with normal gastric epithelial cells. Overexpression of miR204 suppressed GC cells' proliferation, invasion, and migration. It is noteworthy that 5 -FU treatments induced miR-204 expression and suppressed TGF- $\beta$ pathway. By establishment of 5-FU resistant GC cell line, it was revealed that miR-204 was significantly downregulated in 5-FU resistant GC cells, representing mesenchymal features with downregulation of epithelial marker, while mesenchymal markers were upregulated. We identified TGFBR2 as a direct target of miR-204 by Western blot method and luciferase assay in GC cells and tumor samples as well. In addition, overexpression of miR-204 sensitized GC cells to 5-FU in vitro. Xenograft experiments demonstrated that the combination of miR-204 and 5-FU efficiently inhibited tumor growth and improved survival rate of mice as well. Eventually, we illustrated the restoration of TGFBR2 in miR-204 overexpression GC cells, which recovered resistance

\begin{tabular}{ll}
\hline Hui Chen & Department of Gastrointestinal Surgery, \\
& The First Affiliated Hospital of Fujian Medical University, Fuzhou 350005, (China) \\
& Tel. +8618059089988, E-Mail 83801636@163.com
\end{tabular}
\end{abstract}

\section{KARGER}


to 5-FU treatments compared with miR-204 overexpression GC cells. Conclusion: This study describes a miRNA-based therapeutic strategy against 5-FU resistance in GC, contributing to the development of anti-chemoresistance therapeutic agents.

\section{Introduction}

Gastric cancer (GC) is one of the most conventional malignant tumors throughout the world. In addition, it is the most common gastrointestinal malignancy, causing the cancer-related deaths in East Asia [1]. Early detection and effective treatments of GC are essential because of limitation of local invasion and distant metastasis [2]. However, due to intricate complication and achieved chemo-resistance, it is difficult to obtain an effective treatment for higher grade of malignant gastric tumors, and mechanisms associated with chemoresistant GC cells, and metastasis have attracted several research attentions in recent years [3]. In addition, 5-fluorouracil (5-FU) is one of the most frequently used anti-cancer agents for human GC cells [4]. Although numerous studies have reported a precious role of 5-FU in combined treatments with other chemotherapeutic agents, patients with GC have shown progressive 5-FU resistance after initial responding to 5-FU treatment [5].

MicroRNAs (miRNAs) are a class of endogenous, small (18-22 nucleotides) and noncoding RNAs [6]. Moreover, miRNAs can regulate protein expression via post transcriptional repression or by promoting degradation of the targeted mRNAs through direct binding to the 3'-untranslated region (3'-UTR) of their targeted mRNAs [7]. Increasing evidences have indicated that miRNAs play a major role in various types of cancers, including cancer cell, proliferation, differentiation, apoptosis, chemo-sensitivity, metastasis, tumorigenesis, and cellular metabolism $[8,9]$. It has been reported that miR-204 was downregulated in multiple cancers [10-13]. However, the mechanisms of miR-204 in 5-FU sensitivity in GC are still unclear.

Transforming growth factor beta (TGF- $\beta$ ) is overexpressed in multiple tumors, including GC and is negatively correlated with prognosis of patients with GC [14]. TGF- $\beta$ pathway regulates proliferation, differentiation, angiogenesis, wound healing, as well as other functions of different cancers [15]. The epithelial-mesenchymal transition (EMT) is characterized as a fundamental process with increased characteristics of mesenchymal cells and attenuated epithelial features [16]. In addition, aberrant EMT activation in GC is closely associated with carcinogenesis, invasion, metastasis, and tumor progression [17]. It has been caused the loss of E-cadherin expression, while has increased expressions of mesenchymal markers such as N-cadherin, vimentin, and snail, as hallmarks of the EMT process with required enhancement of tumor cell motility [18]. In this study, we investigate the roles of miR-204 in the TGF- $\beta$ induced EMT process in GC cell lines, involving AGS and SGC-7901. The correlation of miR-204, EMT, and 5-FU sensitivity is studied in vitro and in vivo as well. Moreover, the direct target of miR-204 in TGF- $\beta$ pathway is analyzed. The present study plays a vital role in treating GC using a miRNAs-based therapeutic strategy.

\section{Materials and Methods}

\section{Patient samples and cell culture}

The normal gastric epithelial cell line GES-1 and GS cell lines (i.e., AGS, SGC-7901, MKN-45, MGC-803, and BGC-823) were obtained from the Shanghai Institute of Biochemistry and Cell Biology (Shanghai, China) and maintained at The First Affiliated Hospital of Fujian Medical University. Cells were incubated at $37^{\circ} \mathrm{C}$ in a humidified environment containing $5 \% \mathrm{CO}_{2}$. All cells were grown in Dulbecco's modified Eagle's medium (Invitrogen, CA, USA), and supplemented with 10\% fetal bovine serum, $50 \mathrm{IU} / \mathrm{mL}$ of penicillin, and $50 \mu \mathrm{g} /$ $\mathrm{mL}$ of streptomycin. This study was performed in accordance with ethical guidelines under the protocols approved by the Institutional Medical Ethics Review Board of The First Affiliated Hospital of Fujian Medical University (Fuzhou, China), and informed consent was obtained from all patients prior to use of the resected 


\section{Cellular Physiology Cell Physiol Biochem 2018;47:1533-1545 \begin{tabular}{l|l} 
and Biochemistry Published online: June 25, 2018 & $\begin{array}{l}\text { () 2018 The Author(s). Published by S. Karger AG, Basel } \\
\text { www.karger.com/cpb }\end{array}$ \\
\hline
\end{tabular}}

samples. The GC specimens and their adjacent non-tumor mucosa were obtained from 30 patients with GC who underwent gastrectomy at The First Affiliated Hospital of Fujian Medical University (Fuzhou, China), during January 2012 and January 2015. Patient samples were immediately snap-frozen in liquid nitrogen, and stored at $-80^{\circ} \mathrm{C}$ until the start of study. All tissue specimens were pathologically evaluated. No patients received other cancer chemo- or radio-therapy prior to resection.

\section{Antibodies and reagents}

Rabbit monoclonal antibodies against phospho-Smad2 (\#3104), phospho-Smad3 (\#9520), total Smad2 (\#5339), total Smad3 (\#9523), and $\beta$-actin (\#4970) were purchased from Cell Signaling Technology, Inc. (MA, USA). Rabbit polyclonal antibody against TGF beta receptor 2 (TGFBR2) (ab61213) was purchased from Abcam Biotechnology company (Cambridge, UK). Besides, 5-FU was purchased from Sigma-Aldrich Company (MO, USA).

\section{Transfection of microRNA and plasmid DNA}

MiRNA control mimic and miR-204 mimic were synthesized by Gene-Pharma Company (Shanghai, China). TGFBR2 overexpression vector as well as control vector were obtained from Addgene.org (http:// www.addgene.org/). MiRNAs were transfected at $50 \mathrm{nM}$ for 48 hours. Plasmid DNA was transfected at $2 \mu \mathrm{g}$ for 48 hours using Lipofectamine 2000 transfection reagent (Invitrogen, CA, USA) according to the manufacturer's protocol.

\section{Cell proliferation and viability assay}

Cell proliferation was examined by a Quick Cell Proliferation Assay Kit (ab65473; Abcam Co., Cambridge, UK) according to the manufacturer's instructions. The cell viability was measured by 3-(4, 5-Dimethylthiazol2-yl)-2, 5-diphenyltetrazolium bromide (MTT) assay. Besides, cells were plated at a density of $5 \times 10^{4}$ cells/ well in a 96-well plate, and cultured for overnight at $37^{\circ} \mathrm{C}$. The cells were subsequently exposed to various formulations of 5-FU for 48 hours. Cell viability was measured by adding $20 \mu \mathrm{l}$ of MTT dye in $200 \mathrm{ml}$ of phosphate-buffered saline (PBS) (5 mg/ml) per well for another 4 hours at $37^{\circ} \mathrm{C}$ in a humidified chamber containing $5 \% \mathrm{CO}_{2}$. Formazan crystals, which formed due to reduction of dye by viable cells in each well, were dissolved in $150 \mu \mathrm{l}$ of dimethyl sulfoxide and absorbance was measured at $570 \mathrm{~nm}$ using SpectraMax M2 microplate reader (Molecular Devices, LLC., CA, USA). Experiments were undertaken in triplicate as well.

\section{Caspase 3 activity}

Caspase-3 activity was examined by a Caspase 3 Assay Kit (Colorimetric) (ab39401 Abcam Co., Cambridge, UK) according to the manufacturer's instructions.

\section{Real-time RT-PCR}

Total RNA was extracted using TRIzol Reagent (Invitrogen, CA, USA) according to the manufacturer's instructions. The concentration and purity of total RNA were assessed using a NanoDrop ${ }^{\mathrm{TM}} 1000$ Spectrophotometer (NanoDrop Technologies, DE, USA). For detection of miRNA, 500 ng of RNA for each sample was used to synthesize cDNA by TaqMan Advanced miRNA cDNA Synthesis Kit (Thermo Fisher Scientific, MA, USA). Quantification of miRNA expression was performed using TaqMan MicroRNA Assays (Applied Biosystems, CA, USA) according to the manufacturer's

Table 1. Primers for mRNAs used in the present study

\begin{tabular}{lcc}
\hline Genes & \multicolumn{1}{c}{ Forward } & Reverse \\
\hline E-cadherin & 5'-GTCAGTTCAGACTCCAGCCC-3' & 5'-AAATTCACTCTGCCCAGGACG-3' \\
N-cadherin & 5'-GGTGGAGGAGAAGAAGACCAG-3' & 5'-GGCATCAGGCTCCACAGT-3' \\
Snail & 5'-ACCACTATGCCGCGCTCTT-3' & 5'-GGTCGTAGGGCTGCTGGAA -3' \\
Twist & 5'-GGACAAGCTGAGCAAGATTCAGA-3' & 5'-TCTGGAGGACCTGGTAGAGGAA-3' \\
Fibronectin & 5'-CCCACCGTCTCAACATGCTTAG-3' & 5'-CTCGGCTTCCTCCATAACAAGTAC-3' \\
Vimentin & 5'-TCTACGAGGAGGAGATGCGG-3' & 5'-GGTCAAGACGTGCCAGAGAC-3' \\
TGFBR2 & 5'-CCGCTGCATATCGTCCTGT-3' & 5'-AGTGGATGGATGGTCCTATTACA-3' \\
GAPDH & 5'-ACCACAGTCCATGCCATCAC-3' & 5'-TCCACCCTGTTGCTGTA-3'
\end{tabular}




\section{Cellular Physiology Cell Physiol Biochem 2018;47:1533-1545 and Biochemistry Published \begin{tabular}{l|l} 
DOI: 10.1159/000490871 & $\begin{array}{l}\text { C } 2018 \text { The Author(s). Published by S. Karger AG, Basel } \\
\text { www.karger.com/cpb }\end{array}$
\end{tabular}}

Li et al.: miR-204 Sensitizes Gastric Cancer via TGFBR2-Mediated EMT

protocol. Primer for miR-204 is: ttcctttgtcatcctatgcct. U6 was used as an internal control gene as well. For detection of mRNAs, 500 ng of total RNA from each sample was utilized to synthesize cDNA by Sensiscript Reverse Transcription Kit (QIAGEN, Germany). Besides, 500 ng of cDNA was used by SYBR Green Dye for qPCR (Applied Biosystems Inc., CA, USA) based on manufacturer's instructions. Primers for mRNAs were listed in Table 1 . The relative expressions of miRNA or mRNAs were calculated using $2^{-\Delta \Delta c t}$ methods. All reactions were performed in duplicate as well.

\section{Colony formation assay}

AGS parental and 5-FU resistant cells were seeded into a 6-well dish at 500 cells/well for overnight. Cells were then treated with $30 \mu \mathrm{g} / \mathrm{ml}$ of 5-FUfor 2 weeks. Medium containing 5-FU was refreshed each three-day. Cells were stained with crystal violet and colonies were analyzed under a microscope after 14 days.

\section{Transwell invasion Assay}

Cell invasion assays were performed using Matrigel-coated (invasion assay 24-well Transwell inserts with $8 \mu$ m pores) (BD Biosciences, NJ, USA). Briefly speaking, $1 \times 10^{4}$ cells were suspended in $200 \mu \mathrm{l}$ regular medium and were placed into the upper chamber of the insert with or without Matrigel. After 24 hours of incubation, cells remaining on the upper membrane were carefully removed. Cells in lower membrane of each insert were photographed under a microscope and counted using ImageJ software (https://imagej.nih. gov/ij/). All experiments were performed in triplicate.

\section{Wound healing assay}

Cellular motility was assessed by a wound healing assay according to previous descriptions [13]. Briefly, cells with control mimic or miR-204 mimic transfection were plated at a density of $2 \times 10^{4}$ cells/well in a 24-well plate and incubated overnight with $5 \mathrm{ng} / \mathrm{ml} \mathrm{TGF} \beta$. A straight line was then scratched through the attached cells using a sterile tip. Dead cells were removed and images were then captured immediately $(0 \mathrm{~h})$ and at 24 hours using a microscope to record the width of the wound. All experiments were performed in triplicate.

\section{Luciferase reporter assay}

AGS and SGC-7901 cells were plated in a 24-well plate at $1 \times 10^{4}$ cells/well. The cells were cotransfected with $20 \mathrm{nM}$ of either miR-204 mimic or control mimic, 20 ng of either pGL3-TGFBR2-WT 3'UTR or pGL3-TGFBR2-MUT 3'UTR using Lipofectamine 2000 transfection reagent (Invitrogen, CA, USA) for 48 hours. Cells were collected and analyzed using the Dual-Luciferase Reporter Assay System (Promega, WI, USA). Experiments were carried out in triplicate as well.

\section{Western blot analysis}

Cells were lysed by RIPA Lysis and Extraction Buffer (\#89900, Thermo Fisher Scientific, MA, USA). Besides, dithiothreitol (DTT) at final concentration of $20 \mathrm{mM}$ was added into lysis buffer prior use. Cell lysates were centrifuged at $14,000 \mathrm{rpm}$. for 15 minutes to collect supernatant. Protein concentrations were determined by using Bradford assay (Bio-Rad, CA, USA). Equal amounts of each sample were mixed with 2x Loading sample buffer (Bio-Rad, CA, USA) and heated at $95{ }^{\circ} \mathrm{C}$ for 5 minutes. Samples were loaded into $10 \%$ SDS-PAGE followed by transferring proteins onto polyvinylidene difluoride (PVDF) membranes. After blocking with $5 \%$ non-fat milk for one hour at room temperature, membranes were incubated with primary antibodies at $4{ }^{\circ} \mathrm{C}$ for overnight. After washing, the secondary antibodies were goat anti-rabbit, and conjugated to horseradish peroxidase (\#A27036, Thermo Fisher Scientifid, MA, USA). Immuno-stained bands were detected by chemiluminescent method (Pierce, IL, USA).

\section{Xenograft experiments}

The athymic BALB/c nude mice (5-8 week-old) were used in this study. Animal protocols were approved by the Institutional Animal Care and Use Committee animal facility at the University of The First Affiliated Hospital of Fujian Medical University. Mice were implanted subcutaneously into a mouse mammary fat pad (MFP) with $1 \times 10^{7}$ AGS 5-FU resistant cells. When a tumor reached a size of higher than $150 \mathrm{~mm}^{3}$, mice were randomly divided into 4 groups (15 mice per group) as follows: PBS-treated control; 5-FU alone [10 mg/kg 
intraperitoneal (i.p.), twice/week], miR-204 mimic [200 nM intratumoral, twice/week] and 5-FU as well as miR-204 mimic. The rate of mice mortality was daily recorded. The volume of tumor $\left(\mathrm{mm}^{3}\right)$ was calculated by length $\times$ width ${ }^{2} / 2 . \mathrm{CO}_{2}$ chamber was used for euthanasia of mice. All of the animal experiments were complied with both Chinese laws and the guidelines of the Ethics Committee of The First Affiliated Hospital of Fujian Medical University. Experiments were carried out in accordance with the European Communities Council Directive of 24 November 1986 (86/609/EEC).

\section{Target prediction and bioinformatic analysis}

In silico, putative target prediction of miR-204 was performed through analyzing the results of two web server tools: www.Targetscan.org and www.microRNA.org. Expression profiles of TGFBR2 were analyzed from a web-based data-mining platform, www.oncomine.com according to a previous report [19].

\section{Statistical analyses}

The collected data were analyzed by Student's t-tests using GraphPad Prism 5.0 Software (GraphPad Software Inc., CA, USA). Categorical data were analyzed by one-way analysis of variance (ANOVA). $P<0.05$ was considered to be statistically significant.

\section{Results}

MiR-204 is downregulated in GC and suppresses proliferation of cancer cell

There is a growing evidence, that dysregulated miRNAs play pivotal roles in tumorigenesis [6-8]. Moreover, downregulated miRNA-204 is frequently coupled with tumor suppressive activity in multiple tumors [10-13]. In parallel with this evidence, we analyzed the expressions of miR-204 in human gastric tumors, and compared with their adjacent normal tissues. QRTPCR experiments were carried to investigate the expression profiles of miR-204 and it was revealed the reduction of expression of miR-204 in gastric tumors, and compared with their matched normal tissues (see Fig. 1A). The mean expression level of miR-204 was 1.1 and 6.2 in GC and their matched normal tissues, respectively. Afterwards, it was attempted to compare the miR204 expressions in five GC cell lines (i.e., AGS, SGC-7901, MKN-45, MGC803 and BGC-823) with normal human primary gastric epithelial cells. As we expected, miR-204 was downregulated in GC cells compared with normal gastric epithelial cells (see Fig. 1B). To further support the tumor suppressive roles of miR-

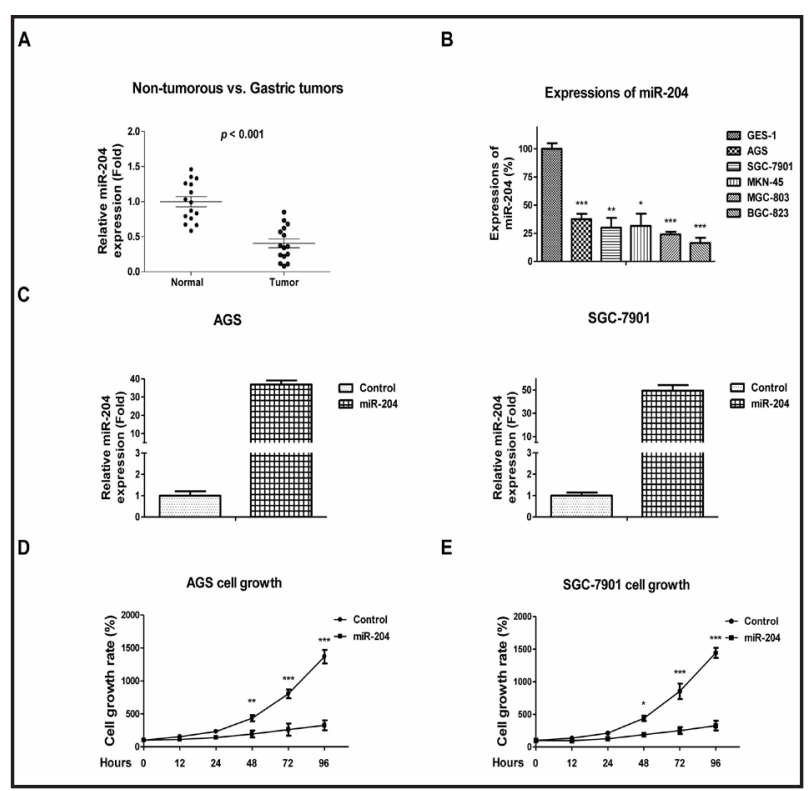

Fig. 1. A tumor suppressive role of miR-204 in GC. (A) Expressions of miR-204 were compared in gastric tumor tissues $(n=15)$ with their matched normal tissues. (B) Expressions of miR-204 were compared in normal human primary gastric epithelial cells with GC cell lines. (C) AGS (left) and SGC-7901 (right) were transfected with control mimic or miR-204 mimic for 48 hours, followed by the measurements of miR-204 expressions by qRT-PCR. Total RNA was subjected to qRT-PCR to analyze the expression level of miR-204. U6 was used as an internal reference for miR-204 expressions. (D) AGC and (E) SGC-7901 cells were transfected with control mimic or miR-204 mimic for 48 hours, followed by the measurements of cell growth by MTT assay. Each sample was analyzed in triplicate. Results are expressed as mean \pm SEM. *: $\mathrm{P}<0.05$; **: $\mathrm{P}<0.01$; *** $\mathrm{P}<0.001$. 
204, we transfected miR-204 precursor or control miRNAs into GC cells AGS and SGC-7901, then the cell proliferation rates were detected with or without miR-204 overexpression, as displayed in Fig. 1C. Accordingly, it was disclosed that cell proliferation rates of GC cell lines AGS and SGC-7901 were remarkably suppressed by exogenous overexpression of miR-204 (see Fig. 1D). Taken together, these results demonstrated that miR-204 plays a tumor suppressive role in gastric tumor.

Suppression of cell
migration, invasion, and
EMT by miR-204 in GC
AGS and SGC-7901
inate from epithelial
originate from epithelial cells and exhibit low motility. Under TGF- $\beta$ induction, cells showed increased migration capacity [20]. It was revealed that migration of AGS and SGC-7901 cells induced by TGF- $\beta$ was inhibited by miR-204 overexpression (see Fig. 2A). Consistently, transwell assay demonstrated a decreased cell invasion potential of GC cells with overexpression of miR-204 under TGF- $\beta$ treatments, as shown in Fig. 2B. It has been reported that the gastric epithelial cancer cells undergo phenotypic switch from epithelial to mesenchymal phenotype with elevated mobility. Furthermore, EMT participates in gastric tumorigenesis and multiple progressions. We then assessed whether miR-204 could regulate EMT process of GC cells. As we expected, GC cells with TGF- $\beta$ treatments showed obviously morphological variations from epithelial phonotype to mesenchymal one, however, this EMT was blocked by overexpression of miR-204, as illustrated in Fig. 2C, reflecting miR-204 involves in the TGF- $\beta$-mediated EMT process of GC cells. Moreover, we observed that E-cadherin, which is an epithelial cell marker, was significantly suppressed by TGF- $\beta$ treatment. Overexpression of miR-204 blocked the suppression of E-cadherin by TGF- $\beta$ (see Fig. 2D). In contrast, other mesenchymal markers (i.e., N-cadherin, vimentin, Snail, Twist, Fibronectin) were significantly induced by TGF- $\beta$ treatments. As a results, pre-overexpression of miR-204 could suppress the TGF- $\beta$-induced mesenchymal markers induction (see Fig. 2D).

\section{MiR-204 is induced by 5-FU treatments}

The above results demonstrated a suppressive function of miR-204 in GC cells. Since recent studies revealed that the EMT process of tumor is highly correlated with chemosensitivity [21], it was attempted to investigate whether the miR-204-mediated EMT blockage associates with chemosensitivity. Accordingly, it was revealed that miR-204 expressions were induced by 5 -FU treatments in AGS and SGC-7901 GC cells (see Fig. 3A), representing that miR-204 may involve in the 5-FU sensitivity of GC cells. Meanwhile, phosphorylations of Smad2/3 in response to TGF- $\beta$ stimulation were markedly inhibited by 5 -FU treatments in AGS and SGC7901 cells, as displayed in Fig. 3B, indicating that 5-FU treatments can inhibit the activation 


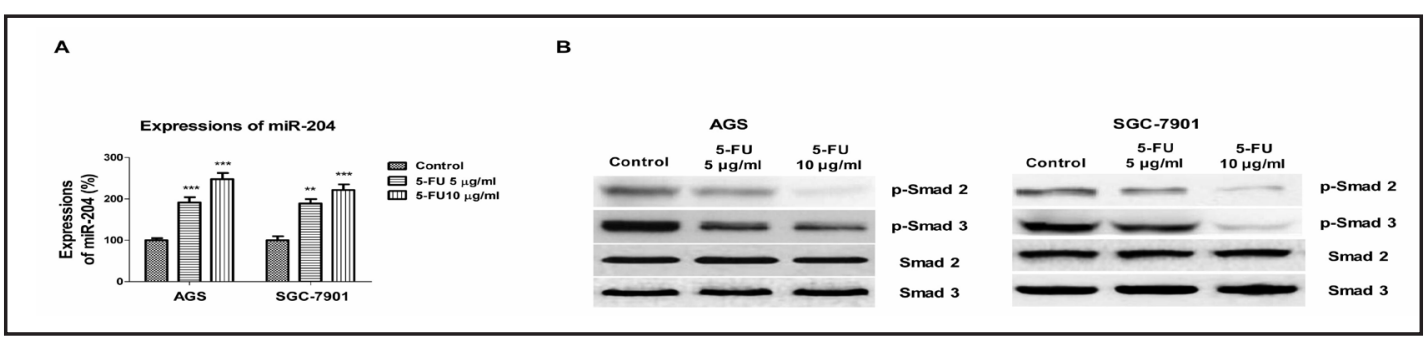

Fig. 3. 5-FU treatment induces miR-204 is induced and suppress TGF- $\beta$ pathway. (A) AGS and SGC-7901 cells were treated with 5-FU at 0, 5, or $10 \mu \mathrm{g} / \mathrm{ml}$ for 48 hours. Expression of miR-204 was measured by qRTPCR. U6 was an internal control. (B) AGS (left) and SGC-7901 (right) cells were treatved with 5-FU at 0, 5, or $10 \mu \mathrm{g} / \mathrm{ml}$ for 48 hours. The phosphorylation of Smad2/3 and total Smad2/3 were analyzed by Western blot method. Each sample was analyzed in triplicate. Results are expressed as mean \pm SEM. ${ }^{* *}$ : $\mathrm{P}<0.01$; ${ }^{* * *}$ : $\mathrm{P}<0.001$.

of TGF- $\beta$ pathway through induction of miR-204 expression in GC cells.

\section{5-FU resistant GC cells display mesenchymal characteristics a $n \quad d$ downregulated $m i R-204$ expression \\ $\mathrm{R}$ e $\mathrm{c}$ e $\mathrm{n} \mathrm{t}$} studies revealed that the acquired 5-FU resistance associates with EMT in colon cancer [22]. To explore the correlation between EMT process and the miR-204 mediated 5-FU sensitivity of GC cells, it was attempted to establish 5-FU resistant GC cell line, originating from AGS GC cell line by repeated subcultures in the presence of stepwise-increases in concentrations of 5-FU [23]. The achieved results, as shown in Fig. 4A, demonstrated that the 5-FU resistant AGS cells were less susceptible to multiple 5-FU treatments than parental cells. The IC50 value of 5-FU resistant AGS cells was increased by 3.1 folds, from 16.33 to $50.5 \mu \mathrm{g} / \mathrm{ml}$, compared with parental cells. Moreover, we performed clonogenic assay as well. AGS parental and 5-FU resistant cells were treated with $30 \mu \mathrm{g} / \mathrm{ml} 5$-FU for two weeks, and they could notably increase cell survival clones against 5-FU, which was observed in AGS 5-FU resistant cells, as displayed in Fig. 4B. As we expected, the 5-FU resistant cells demonstrated a mesenchymal-like phenotype (see Fig. 4C), reflecting that the EMT process of GC cells may contribute to 5-FU resistant. To determine 
whether these morphological variations were associated with EMT, the expressions of epithelial and mesenchymal markers were examined. Consistent with morphological variations, the epithelial marker, E-cadherin was significantly downregulated, while the mesenchymal marker N-cadherin, Fibronectin, Twist, and Snail were markedly upregulated in 5-FU resistant cells compared with parental cells (see Fig. 4D). To investigate whether miR-204 associates with 5-FU resistance and the acquired mesenchymal-like characteristics, the expression of miR-204 in AGS parental and 5-FU resistant cells was compared, and it was disclosed that miR-204 was significantly downregulated in 5-FU resistant cells (see Fig. 4E). Taken together, these results represent that the acquired drug resistance may associate with EMT cellular process and downregulation of miR-204.

\section{MiR-204 directly targets TGFBR2 in GC}

The achieved resultsindicated a correlation between the 5-FU induced mir-204 expression and TGF- $\beta$ pathway. In the nextstep, it was attempted to assess whether miR-204 could directly target TGF- $\beta$ pathway. To seek potential targets of miR-204, a bioinformatics analysis was undertaken through two online miRNAs target prediction programs TargetScan.org (http:// www.targetscan.org/) and microRNA.org (http://www.microrna.org), which could predict a single miRNA-responsive element containing a conserved 8-mer nucleotide at positions 2324-2331 of TGFBR2 3'UTR as a targeted miR-204 (see Fig. 5A). Moreover, the binding sites of miR-204 on TGFBR2 3'UTR were conserved in multiple species, as illustrated in Fig. 5B. To verify this potential target of miR-204, GC cells were transfected with control miR-204 or miR204 precursor. The results of Western blot analysis showed that overexpression of miR-204 $\mathrm{re} \mathrm{m}$ a r k a b ly s u p p r e s s ed

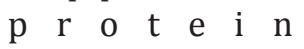
expressions of TGFBR2 (see Fig. 5C). To assess whether miR-204 could bind to the predicted 3'UTR region of TGFBR2, we inserted a fragment of around 400 base pairs of the TGFBR2 3'UTR, containing miR204 binding $\mathrm{s}$ e q u e $\mathrm{n} \mathrm{c}$ e $\mathrm{s}$ downstream of the luciferase coding regions. As we expected, co-transfection of miR-204 with luciferase vector, containing wild type TGFBR2

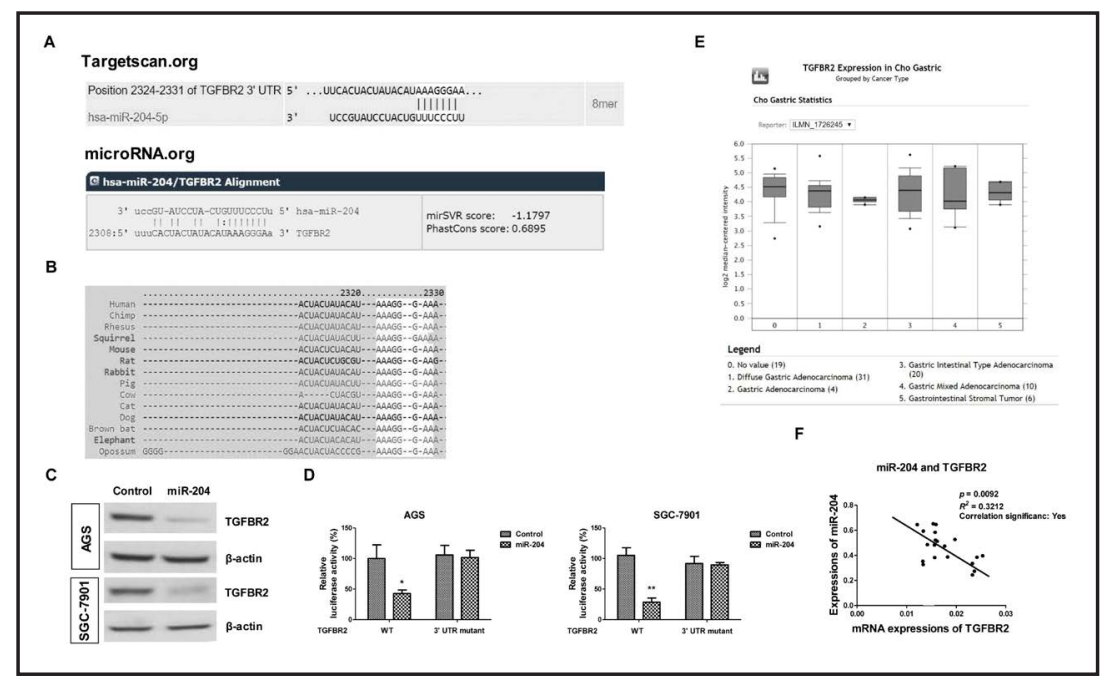

Fig. 5. MiR-204 directly targets TGFBR2. (A) Illustration of the TGBFR2 3'UTR and the seed sequence of miR-204 shows the predicted binding sites on the 3'UTR of TGFBR2 mRNA by Targetscan.org and microRNA.org (http://www.targetscan.org/, http://www.microrna.org). (B) Conserved binding sites of miR-204 on TGBFR2 3'UTR in multiple species analyzed by Targetscan.org. (C) Western blotting of TGFBR2 protein levels in AGS (upper) and SGC-7901 (lower) cells with or without miR-204 overexpression. $\beta$-actin was an internal control. (D) Overexpression of miR-204 decreases TGFBR2 3'UTR luciferase activity. AGS (left) and SGC-7901 (right) cells were co-transfected with $2 \mu \mathrm{g}$ of luciferase vector, containing either wild type TGFBR2 3'UTR or binding site mutant TGFBR2 3'UTR along with $50 \mathrm{nM}$ miR-204 mimic for 48 hours. Cells were lysed and assessed for luciferase activity. (E) TGFBR2 expressions in gastric cancer cells were analyzed from oncomine. org (https://www.oncomine.org/). (F) Correlation between miR-204 and mRNAs of TGFBR2 in gastric tumor tissues was analyzed. mRNA expressions of TGFBR2 were detected by qRT-PCR. GAPDH was an internal control. Results are expressed as mean \pm SEM. *: $\mathrm{P}<0.05$; **: $\mathrm{P}<0.01$. 
3'UTR significantly reduced the luciferase activity when compared with co-transfection of control miRNA (see Fig. 5D). Additionally, this inhibitory effect was not proved in the presence of co-transfection of miR-204 with binding sites mutated 3'UTR of TGFBR2 (see Fig. 5D). By analyzing the TGFBR2 expression profiles in human gastric tumors using an online oncology bioinformatics database, Oncomine.org (https://www.oncomine.org/), it was revealed that TGBFR2 was downregulated in multiple human gastric tumors, as shown in Fig. 5E. To evaluate whether miR-204 could target TGFBR2 in human gastric tumors, we analyzed the correlation between mRNA expressions of TGFBR2 and miR-204. Consistently, a strong negative correlation between miR-204 and TGFBR2 expressions $\left(R^{2}=0.3212, P<\right.$ 0.01 ) was found in human gastric tumor samples; the higher expression of TGFBR2 was detected in lower miR-204 expressing tumors (see Fig. 5F). Consequently, these results demonstrate that the oncogenic TGFBR2 is a direct target of miR-204, a tumor suppressive miRNA in human GC.

\section{Overexpression of miR-204 sensitizes GC cells to 5-FU in vitro and in vivo}

The results revealed that miR-204 is a tumor suppressive miRNA in GC. As the miR-204/ TGFBR2 could regulate the EMT process of GC cells, it was attempted to investigate whether it could modulate the cell sensitivity to 5-FU treatments. The GC cells were treated with or without miR-204 overexpression with 5-FU. In two GC cell lines, AGS and SGC-7901 with miR204 overexpression consistently showed decreased IC50 values of 5-FU (see Figs. 6A and 6B). Moreover, the increased activity of Caspase-3 was detected (see Figs. 6C and 6D) in miR-204 overexpressing cells under 5-FU treatments compared with control miRNAs. These results demonstrate that miR-204 sensitizes GC cells to 5-FU through induction of apoptosis pathway. Next, it was attempted to assess whether miR-204 affects the 5-FU sensitivity of GC cells in a mouse xenograft model. performed number of experiments were then performed to explore whether the combination of miR-204 mimic with 5-FU chemotherapy could significantly increase the survival rate and inhibit tumor growth in vivo. Nude mice were inoculated with

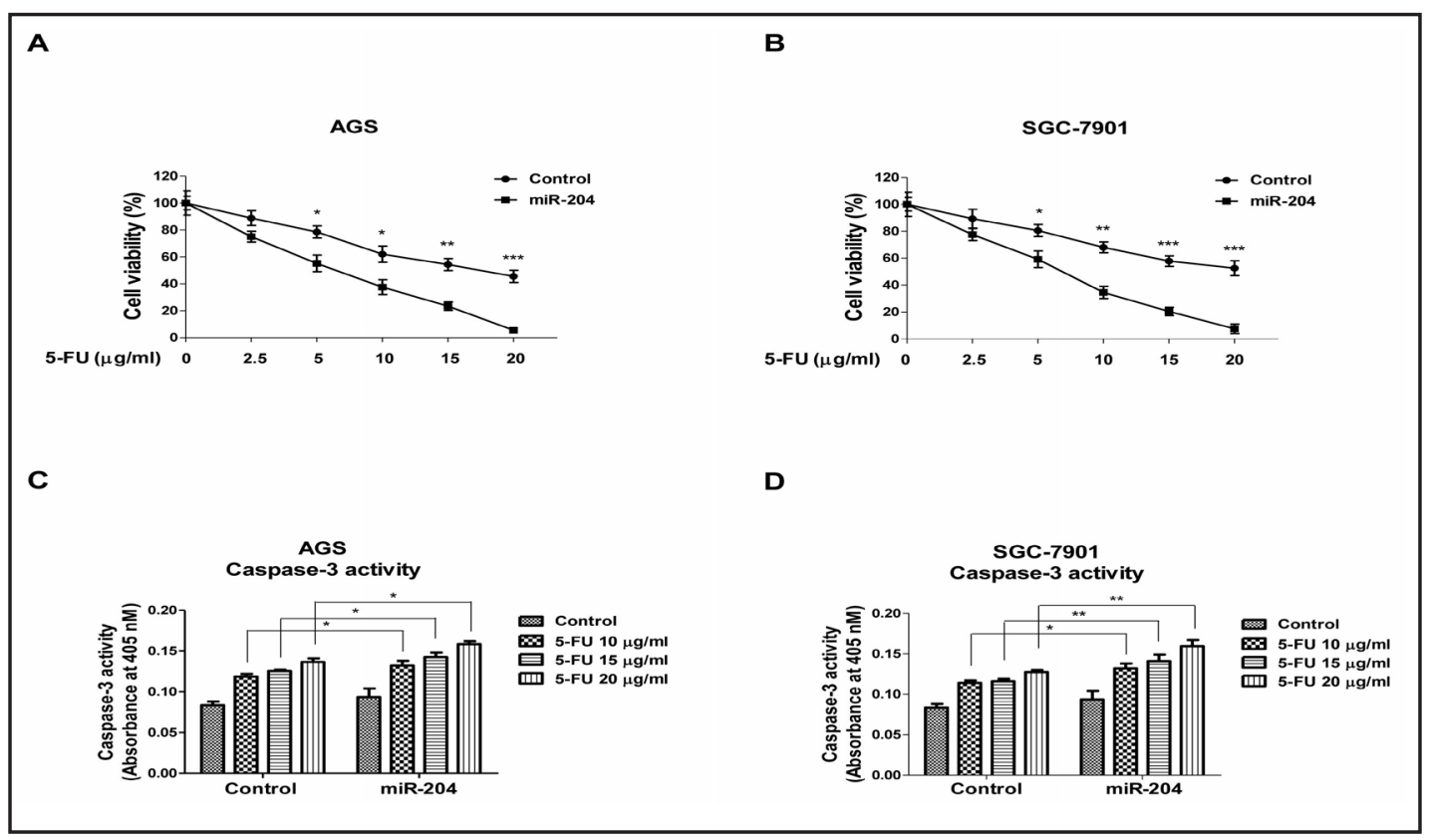

Fig. 6. Overexpression of miR-204 sensitizes GC cells to 5-FU. (A) AGS and (B) SGC-7901 cells were transfected with control mimic or miR-204 mimic for 48 hours. Cells were treated with 5-FU at 0, 2.5, 5, 10,15 , or $20 \mu \mathrm{g} / \mathrm{ml}$ for 48 hours. The cell viability was measured by MTT assay. (C) AGS and (D) SGC-7901 cells were transfected with control mimic or miR-204 mimic for 48 hours, cells were treated with 5-FU at 0, 10,15 , or $20 \mu \mathrm{g} / \mathrm{ml}$ for 48 hours. Caspase activity assay was then performed. Each sample was analyzed in triplicate. Results are expressed as mean \pm SEM. *: $\mathrm{P}<0.05$; **: $\mathrm{P}<0.01$; ***: $\mathrm{P}<0.001$. 
AGS 5-FU resistant cells into the MFPs for the establishment of xenograftmouse model. Mice were then treated with PBS control, 5-FU alone, miR-204 mimic alone or 5-FU plus miR-204 mimic via intraperitoneal injection weekly. It was disclosed that most of the animals died within 2 months after treatments with control or 5-FU alone, while the combination of miR204 mimic and 5-FU dramatically achieved a prolonged survival time of mice (see Fig. 7A). In addition, it was revealed that miR204 mimic treatment could inhibit the tumor

growth in vivo (see Figs. 7B and 7C). Moreover, tumor sizes of mice that received treatment with 5-FU alone as well as none treatment were remarkably larger than that of miR-204 mimic injection and 5-FU plus miR-204 treatments, as displayed in Figs. 7B and 7C. In addition, the dimensions of tumor in mice with the combination of miR-204 and 5-FU were significantly smaller than that of miR-204 mimic treatment (see s. 7B and 7C), reflecting that the miR-204 plus 5-FU treatments might be an effective therapeutic strategy to treat 5-FU resistant patients. To verify the in vitro results, that miR-204 directly targets TGFBR2 and 5-FU treatments could inhibit TGFBR2, we analyzed the mRNA expressions of TGFBR2 in the tumors in mice. As we expected, the TGFBR2 expressions in the tumors in mice with 5-FU alone, miR-204 alone or the combination of 5-FU and miR-204 were considerably downregulated compared with the tumors in mice with non-treatment (see Fig. 7D). Among them, mRNA expressions of TGFBR2 with 5-FU plus miR-204 treatment was lower in tumors in mice than 5-FU alone or miR-204 alone, as illustrated in Fig. 7D. In summary, the achieved results indicate that combination of miR-204 with 5-FU chemotherapy presented significantly a superior outcome in the suppression of development of xenograft mouse model through targeting TGFBR2.

\section{Restoration of TGFBR2 decreases 5-FU sensitivity of GC cells}

To consolidate the roles of the miR-204-targeted TGFBR2 in 5-FU sensitivity, we performed rescue assays. The complete open reading frame of TGFBR2 genes were cloned into the pcDNA3 expression vector. Western blot analysis results showed that transfection of TGFBR2 overexpression vector into miR-204 overexpressing GC cells could restore the protein expression of TGFBR2 (see Fig. 8A). Cells with or without restoration of TGFBR2 were treated with 5 -FU at $0,2.5,5,10,15$, or $20 \mu \mathrm{M}$. As we expected, restoration of TGFBR2 in miR-204 overexpression GC cells resulted in increased resistance to 5-FU treatments in comparison with controls (see Fig. 8B). In addition, the activities of Caspase- 3 were decreased in TGFBR2 restored cells under 5-FU treatments (see Fig. 8C). Consequently, these results indicate that TGFBR2 is a direct effector of miR-204 in response to 5-FU treatment and its forced expression rescued the miR-204-mediated 5-FU sensitivity.

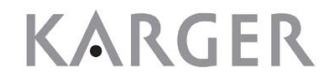




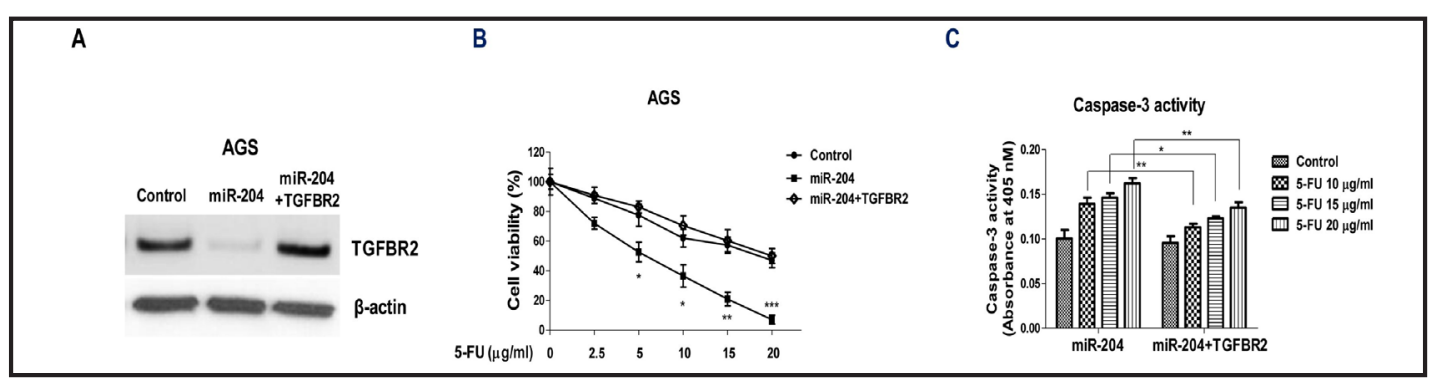

Fig. 8. Restoration of TGFBR2 decreases 5-FU sensitivity of GC cells. (A) AGS cells were transfected with control mimic, miR-204 mimic or miR-204 mimic plus TGFBR2 overexpression vector. Protein expressions of TGFBR2 were detected by Western blot technique. $\beta$-actin was used as an internal control. (B) AGS cells were transfected with control mimic, miR-204 mimic, or miR-204 mimic plus TGFBR2 overexpression vector. Cell were treated with 5 -FU at $0,2.5,5,10,15$, or $20 \mu \mathrm{g} / \mathrm{ml}$ for 48 hours. The cell viability was detected by MTT assay. (C) AGS cells were transfected with miR-204 mimic or miR-204 mimic plus TGFBR2 overexpression vector. Cells were treated with 5 -FU at $0,10,15$, or $20 \mu \mathrm{g} / \mathrm{ml}$ for 48 hours. Caspase-3 activity was measured. Each sample was analyzed in triplicate. Results are expressed as mean \pm SEM. *: P<0.05; **: $\mathrm{P}<0.01$; **: $\mathrm{P}<0.001$.

\section{Discussion}

In the present study, the expression of miR-204 in human gastric tumor cells and their matched normal gastric tissues was detected. It is widely studied that the aberrant expressions of microRNAs play a significant role in tumorigenesis and development [6-9]. During tumorigenesis, a group of microRNAs are downregulated in cancer tissues. These types of microRNAs are taken into account as tumor suppressive miRNAs, which negatively regulate oncogenes that promote cancer cell proliferation, differentiation, migration and chemoresistance [6-9]. Currently, miR-204 has been reported to be markedly downregulated in various tumor tissues. a previous study was performed regarding genome wide analysis to interrogate miRNA expression profile of gastric tumor specimens compared with their matched peritumoral tissues; consequently, they found that 4 miRNAs (miR-204, miR-148a, miR-31, miR-375) were selectively downregulated in tumor tissues and ectopic expression of miR-204 inhibited colony formation, migration and tumor engraftment of GCs [24]. Consistently, the results achieved in the present study demonstrated that miR-204 was downregulated in human gastric tumor samples compared with their matched normal gastric tissues. In addition, the overexpression of miR-204 has suppressed proliferation, migration, invasion, and the TGF- $\beta$ induced EMT of GC cells. Therefore, the obtained data demonstrate that microRNA-204 is a tumor suppressive miRNA in GC.

Although the improvements in quality of the combined surgery and chemotherapy treatments were achieved, prognosis of GC was poorly developed, especially for tackling advanced and disseminated gastric tumors. 5-FU is one of the most frequently used anticancer agents in the treatment of different types of cancers, including colorectal cancer, breast cancer, colorectal cancer, and GC [4]. Currently, 5-FU in combination with other chemotherapeutic agents has demonstrated improved response and survival rate in GC [4]. However, chemoresistance of 5-FU restricts the effectiveness of GC treatment. Hence, it is vital to investigate the relevant mechanisms and identify therapeutic targets that involve in 5-FU resistance.

A number of mechanisms are responsible for drug resistance, involving activation of Hedgehog (Hh), Notch, and Wingless (Wnt) signaling pathways [25, 26]. Moreover, the EMT process of cancer cells is known as a significant regulator for cancer cells migration, invasion, and chemoresistance. Recent studies have reported that 5 -FU resistance associates with EMT in colon cancer cells, that 5-FU resistant cells have showed mesenchymal features compared 
with sensitive colon cancer cells [22]. In addition, two previous studies have reported that epithelial-to-mesenchymal transition contributes to chemoresistance of lung cancer [27] and pancreatic cancer [28], reflecting that the acquired EMT cellular process might be a therapeutic target for development of anti-chemoresistance drugs. The results obtained in the present study demonstrated that 5-FU resistant GC cells have shown mesenchymal feathers with upregulation of mesenchymal markers, however, downregulation of epithelial marker, E-cadherin, supports the previous findings.

In addition, in this study, it was revealed that miR-204 could direct targeted TGFBR2, which is an important upstream regulator for the TGF- $\beta$-modulated EMT process. Further evidence shows that TGF- $\beta$ is up-regulated in human malignancies, including GC [14]. Besides, TGF- $\beta$ pathway plays a critical role in diverse biological processes, involving proliferation, differentiation, angiogenesis, invasion, and migration [15]. A previous research has shown that aberrant activation of EMT is closely associated with carcinogenesis, invasion, metastasis, and tumor progression of human cancers [17]. Numerous studies have reported that several molecules might affect EMT of cancer cells, including TGF- $\beta$, epithelial growth factor (EGF), and platelet derived growth factor (PDGF) [29, 30]. Moreover, we observed that miR-204 was significantly downregulated in 5-FU resistant GC cells, providing a correlation between 5-FU sensitivity, 5-FU, and the TGFBR2-modulated EMT. Furthermore, overexpression of miR-204 sensitized 5-FU resistant cells from in vitro and in vivo xenograft experiments, reflecting that the combination of miR-204 with 5-FU might be an effective therapeutic strategy for overcoming 5 -FU resistance in patients with GC.

In conclusion, the present study demonstrates that an EMT phenotype and decreased miR-204 associate with 5-FU resistance in GC cells. Overexpression of miR-204 sensitized 5 -FU resistant GC cells through suppression of the TGF- $\beta$ mediated EMT signaling pathway. These findings may present a novel clinical therapeutic approach for the treatment of GC with acquired 5- FU resistance.

\section{Acknowledgements}

This study was supported by the National Key Clinical Specialist Construction Programs of China, Fujian Provincial Natural Science Foundation Projects (Grant Nos. 2016J01447), and Fujian Provincial Medical Innovation Project (Grant No.2015-CX-23).

\section{Disclosure Statement} article.

The authors declare there is no conflict of interests regarding the publication of this

\section{References}

$\rightarrow 1$ Fontana E, Smyth EC: Novel targets in the treatment of advanced gastric cancer: a perspective review. Ther Adv Med Oncol 2016;8:113-125.

-2 Newton AD, Datta J, Loaiza-Bonilla A, Karakousis GC, Roses RE: Neoadjuvant therapy for gastric cancer: current evidence and future directions. J Gastrointest Oncol 2015;6:534-543.

3 Shi WJ, Gao JB: Molecular mechanisms of chemoresistance in gastric cancer. World J Gastrointest Oncol 2016;8:673-681.

4 Longley DB, Harkin DP, Johnston PG: 5-fluorouracil: mechanisms of action and clinical strategies. Nat Rev Cancer 2003;3:330-338.

5 Holohan C, Van Schaeybroeck S, Longley DB, Johnston PG: Cancer drug resistance: an evolving paradigm. Nat Rev Cancer 2013;13:714-726.

-6 Lin S, Gregory RI: MicroRNA biogenesis pathways in cancer. Nat Rev Cancer 2015;15:321-333. 


\section{Cellular Physiology Cell Physiol Biochem 2018;47:1533-1545 \begin{tabular}{l|l} 
DOI: 10.1159/000490871 & Ond Biochemistry 2018 The Author(s). Published by S. Karger AG, Basel \\
www.karger.com/cpb
\end{tabular}}

Li et al.: miR-204 Sensitizes Gastric Cancer via TGFBR2-Mediated EMT

7 Rupaimoole R, Slack FJ: MicroRNA therapeutics: towards a new era for the management of cancer and other diseases. Nat Rev Drug Discov 2017;16:203-222.

8 Hayes J, Peruzzi PP, Lawler S: MicroRNAs in cancer: biomarkers, functions and therapy. Trends Mol Med 2014;20:460-469.

9 Yin Y, Li J, Chen S, Zhou T, Si J: MicroRNAs as diagnostic biomarkers in gastric cancer. Int J Mol Sci 2012;13:12544-12555.

10 Flores-Perez A, Marchat LA, Rodriguez-Cuevas S, Bautista-Pina V, Hidalgo-Miranda A, Ocampo EA, Martinez MS, Palma-Flores C, Fonseca-Sanchez MA, Astudillo-de LVH, Ruiz-Garcia E, Gonzalez-Barrios JA, PerezPlasencia C, Streber ML, Lopez-Camarillo C: Dual targeting of ANGPT1 and TGFBR2 genes by miR-204 controls angiogenesis in breast cancer. Sci Rep 2016;6:34504.

11 Wu H, Liang Y, Shen L, Shen L: MicroRNA-204 modulates colorectal cancer cell sensitivity in response to 5-fluorouracil-based treatment by targeting high mobility group protein A2. Biol Open 2016;5:563-570.

12 Xia Y, Zhu Y, Ma T, Pan C, Wang J, He Z, Li Z, Qi X, Chen Y: miR-204 functions as a tumor suppressor by regulating SIX1 in NSCLC. FEBS Lett 2014;588:3703-3712.

13 Yu CC, Chen PN, Peng CY, Yu CH, Chou MY: Suppression of miR-204 enables oral squamous cell carcinomas to promote cancer stemness, EMT traits, and lymph node metastasis. Oncotarget 2016;7:20180-20192.

14 Pickup M, Novitskiy S, Moses HL: The roles of TGFbeta in the tumour microenvironment. Nat Rev Cancer 2013;13:788-799.

15 Neuzillet C, Tijeras-Raballand A, Cohen R, Cros J, Faivre S, Raymond E, de Gramont A: Targeting the TGFbeta pathway for cancer therapy. Pharmacol Ther 2015;147:22-31.

16 Kalluri R, Weinberg RA: The basics of epithelial-mesenchymal transition. J Clin Invest 2009;119:14201428.

17 Peng Z, Wang CX, Fang EH, Wang GB, Tong Q: Role of epithelial-mesenchymal transition in gastric cancer initiation and progression. World J Gastroenterol 2014;20:5403-5410.

18 Lamouille S, Xu J, Derynck R: Molecular mechanisms of epithelial-mesenchymal transition. Nat Rev Mol Cell Biol 2014;15:178-196.

19 Rhodes DR, Yu J, Shanker K, Deshpande N, Varambally R, Ghosh D, Barrette T, Pandey A, Chinnaiyan AM: ONCOMINE: a cancer microarray database and integrated data-mining platform. Neoplasia 2004;6:1-6.

20 Li LC, Peng Y, Liu YM, Wang LL, Wu XL: Gastric cancer cell growth and epithelial-mesenchymal transition are inhibited by gamma-secretase inhibitor DAPT. Oncol Lett 2014;7:2160-2164.

21 Du B, Shim JS: Targeting Epithelial-Mesenchymal Transition (EMT) to Overcome Drug Resistance in Cancer. Molecules 2016;21:

22 Liu Y, DU F, Zhao Q Jin J, Ma X, Li H: Acquisition of 5-fluorouracil resistance induces epithelial-mesenchymal transitions through the Hedgehog signaling pathway in HCT-8 colon cancer cells. Oncol Lett 2015; 9:26752679.

23 Chung YM, Park S, Park JK, Kim Y, Kang Y, Yoo YD: Establishment and characterization of 5-fluorouracilresistant gastric cancer cells. Cancer Lett 2000;159:95-101.

24 Sacconi A, Biagioni F, Canu V: miR-204 targets Bcl-2 expression and enhances responsiveness of gastric cancer . Cell Death Dis,2012;3:423-435.

-25 Chu H, Zhang A H, Han Y: Metabolomics and its potential in drug dscovery and development from TCM. World J Tradit Chin Med, 2015;1:26-32.

-26 Yang L, Xie G, Fan Q, Xie J: Activation of the hedgehog-signaling pathway in human cancer and the clinical implications. Oncogene 2010;29:469-481.

-27 Fischer KR, Durrans A, Lee S, Sheng J, Li F, Wong ST, Choi H, El RT, Ryu S, Troeger J, Schwabe RF, Vahdat LT, Altorki NK, Mittal V, Gao D: Epithelial-to-mesenchymal transition is not required for lung metastasis but contributes to chemoresistance. Nature 2015;527:472-476.

-28 Zheng X, Carstens JL, Kim J, Scheible M, Kaye J, Sugimoto H, Wu CC, LeBleu VS, Kalluri R: Epithelial-tomesenchymal transition is dispensable for metastasis but induces chemoresistance in pancreatic cancer. Nature 2015;527:525-530.

29 Matsuoka J, Yashiro M, Doi Y: Hypoxia Stimulates the EMT of Gastric Cancer Cells through Autocrine TGF $\beta$ Signaling. Plos One 2013; 8:62310-62319.

-30 Xiang X J, Deng J, Liu Y W: MiR-1271 Inhibits Cell Proliferation, Invasion and EMT in Gastric Cancer by Targeting FOXQ1. Cell Physiol Biochem 2015;36:1382-1394. 